\title{
Student Learning Centric Methodology: An aid to Innovative Teaching and Learning Process
}

\author{
M.Rithvik ${ }^{1}$, Dr.D. Haritha ${ }^{2}$ \\ ${ }^{1}$ Assistant Professor Department of CSE, SRK Institute of Technology, Enikepadu, Vijayawada \\ ${ }^{2}$ Professor, Department of CSE, SRK Institute of Technology, Enikepadu, Vijayawada \\ ${ }^{1}$ rithvikmadugula@gmail.com, ${ }^{2}$ harithadasari@rediffmail.com
}

\begin{abstract}
Teaching is a noble profession that contributes to the Nation Prosperity and with rapid advancing technology, it is necessary for a professional teacher to understand, inculcate, evaluate, examine and enhance the standards of teaching and learning using the available state-of-art technology yet keeping in view the abilities and limitations of both teachers and learners. While teaching and learning seemingly look dichotomous, they are really intertwined. Collaborative aspects of a complex process of learning are to be shaped by both teacher and student. This paper describes a Student Learning Centric (SLC) and an Analogous Learning methodology to create vibrant and active learning process. In this paper the OSI layers of a Communication Network are interpreted with a Bike Race example so that the functionality of each layer is more effectively understood by the students. This methodology emphasises the need for introducing the new concepts with appropriate real time scenario for effective and active learning.
\end{abstract}

Keywords: Active Teaching and Learning, Inquisitive Learning, Analogous Learning, Learning Assessment

\section{Introduction}

Teaching and learning are intertwined with each other. Domain Knowledge with insights into the learning process can help the teachers to create active classrooms. Today, the digital world is fast penetrating the education and skills domain. Teachers need to acquire contemporary knowledge and also new innovative tools of teaching that are appropriate for the student generation $\mathrm{Z}$. The teachers need to shift the teacher centric class rooms to student learning centric classrooms added with appropriate learning assessment which is essence of outcome-based education.

The words of A.P.J Abdul Kalam, "Learning gives creativity, creativity leads to thinking, thinking provides knowledge and knowledge makes you great", bring realization among the learner community that learning must be an enthusiastic element in all areas to gain knowledge. Learning byheart should be changed to Learning by heart especially in the student and teachers' point of view. "Teaching without learning is just talking" sums up the inactive learning element in conventional teaching. The professional efficacy in all professions in the society depends on effective learning and applying the knowledge and this onus rests on the teachers.

It signifies the teacher's responsibility to make learning in classroom active, fruitful and help in progressive learning. This can happen while exploring the requisite strategic teaching learning process especially in Engineering Education, as most of the Engineering students are not willing to listen until and unless the classroom is active and the session is active. The technological advents made especially in the $21^{\text {st }}$ century aid the teachers in bringing creative outcomes in the learning strategies which in turn promotes effective learning. In order to instil better learning a teacher must be specific about formulating the course objectives that meet the course outcomes. Imagination, Innovation and Introspection are the three quintessential elements that the teacher can strategise to match his/her mission with students' vision. These three elements are the essential qualities/abilities for the industrial growth in the country and play a major role in attaining the new startup/enterprises by the young budding entrepreneurs.

In conventional teaching often the learning objectives overshadow and overlook the actual expected learning outcomes especially in curriculum where the teachers' degree of freedom is limited. The learning objectives play a key role in the teaching domain because these objectives interpret the basis of what to be taught in a classroom. However, if the teacher were to visualize and design learning objectives in a wider perspective which may ignite students' creativity, then the learning outcomes would also have far reaching implications which in turn will benefit the society.

Of course, the teacher has to visualize the learning objectives with empathy for each individual student's vision and grasping abilities. This is not as simple as discussed in the theory the teacher practically needs to bring the creative minds of the classroom in taking these learning objectives and evaluate the outcomes.

This paper illustrates an Analogous Learning technique that enables a student in hands-on learning that aids in new explorations for application. Section 2 discusses the processes that can provide more insights to the teachers on learner-centred teaching and its advantages over conventional teaching. In Section 3, a student learning centric methodology that embodies analogous and inquisitive and learning assessment. In Section 4 the overall 
effectiveness of the methodology is assessed by the amount of active learning.

\section{Literature Review}

Current Education system faces several socio economic and technological challenges and is to be addressed. This requires a high degree of flexibility and adaptability in the education system to meet these challenges. The researchers also stress on the need for a greater degree of inculcation of imaginative and creative thinking in learning, based on broader perception of generation $\mathrm{Z}$ student's mindset, abilities and communication skills. New approaches are needed to find a gateway to build motivational spirit and self-esteem of the students for an inclusive and heterogeneous classroom. Study of evolving classroom methodology creates a scope for devising new effective teaching techniques.

\section{Conventional Teaching:}

The conventional teaching method includes preparation of lesson plan with Course Outcomes (CO) and teaching methodology for respective course contents. The course outcomes are defined based on Bloom's taxonomy. The course delivery is based on conventional teaching aids like chalk and board, presentation slides. The assessment pattern includes a continuous assessment and also semester end evaluation. The continuous assessment includes periodical tests, assignment, tutorial and quizzes. The end semester evaluation includes a written examination comprising the course syllabus. The conventional teaching learning framework is as depicted in Figure 1.

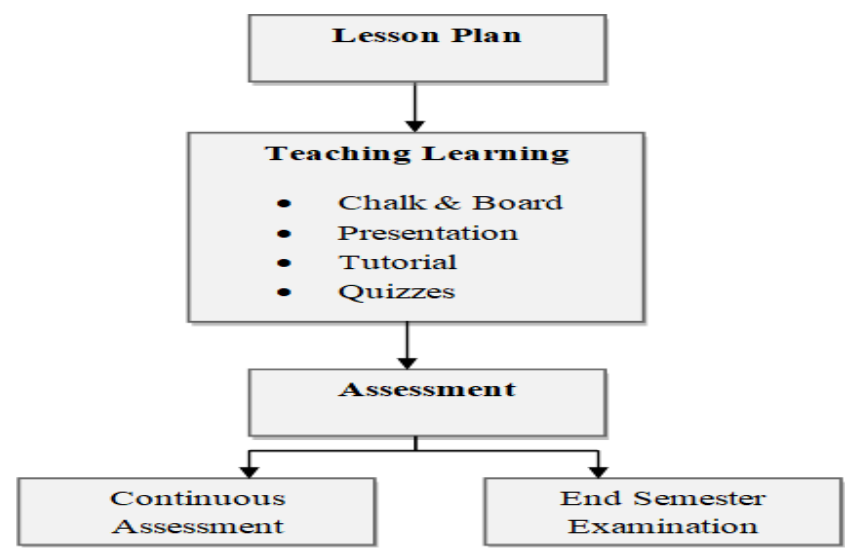

Fig 1: Framework of Conventional Teaching Student- Learning Centred Teaching:

In 1997 Catalano et al,, projected the necessity for transformation from teacher centred to student centred engineering education [1]. Student learning centred teaching is a teaching process which focuses on student's ability to think out of the box, stimulate their higher order thinking skills and further towards hand-on activity. Through this active learning technique, students get the ability to turn ideas into action by integrating creativity, innovation and risk-taking as well as the ability to plan and manage projects in order to meet the learning objectives [1].

Learning can be in dualism of either student-centred learning or teacher-centred learning. However, in reality, student centred learning contributes more to learning continuum as the student plays vital role as depicted in Figure 2

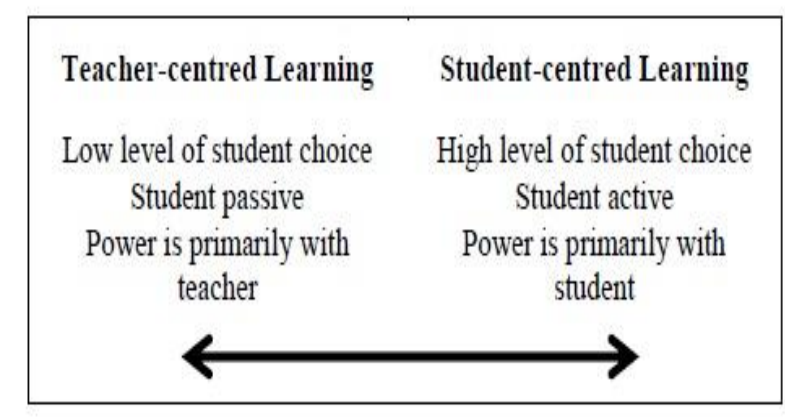

Fig 2: Teacher and Student-centred learning

This paper [1] illustrated that percentage of active listening students is more in the student-centred learning than teacher centred learning. This is possible because the student centric learning awakens the self-esteem and motivates the students making learning joyful.

Amal Abdelsattar et al., emphasized that the engineering students learn better if the students were made to participate, act, react, and reflect, rather than by watching and listening to lectures. Various strategies of active learning approach, their advantages and challenges of implementing them as an integral part of engineering education were discussed [5].

Ricardo et al., opined that in a challenge based learning, a disruptive active learning technique helps students to develop skills and improve their competency. Challenge makes the students out of their comfort zone and helps to think of the solution of problems more creatively [6]. Divya Asok et al established a fact that Active Learning Environment (ALE) coupled with Information and communication technologies (ICT) can promote higher order thinking skills in students [7]. Magana et al., also emphasized that independent learners may benefit more from active learning methods based on their study on engineering learners' preferences for learning methods with various degrees of activity [8].

Now the focus is being shifted towards Enterprise Education. Enterprise education enhances students' capability to enquiry based deep learning, and contributes to make them successful Graduates equipped to face the challenges of their future careers. It focuses on students' responsibilities in learning, collectiveness, and multidimensional life skills. Enterprise education aims to produce scholars with entrepreneurial mindsets, notable aptitude in learning, sharpened abilities to grasp 
opportunities and venture into business field [2]. However, the implementation, awareness and mastery skills in deepening entrepreneurial mindsets among University students are still lagging behind.[3].

We focussed on Analogous learning technique keeping in view the inclusive nature of the classroom to promote inquisitiveness, comparative study and creative thinking and is discussed in the next section.

\section{Student Learning Centric Methodology: An Analogous Learning Technique}

Teaching and learning that constitutes a classroom requires an analogy to understand the learner's perspective and make respective changes in delivering lecture in the classroom. In this regard active learning aided with Elearning plays a key role especially in Engineering Education in bringing teaching and learning together and thus transforming a conventional classroom into an active learning classroom.

There is a large gap existing between student learning centric teaching and conventional way of teaching. Analogous learning is learning from examples and situations that are different from but share similar attributes. Analogous learning is proven to be an effective method in active learning. We present a methodology that emphasizes the need for enhancing the imaginative power of students by giving a real time scenario, drawing the conclusions from the given example to understand and correlate with the new topic being introduced. This methodology or the experiment described demonstrates the effectiveness of active learning over the conventional teaching. This comparative methodology is aligned with the learning objectives and outcomes in framing a new strategy in the teaching learning process in order to evaluate, examine, explore and imbibe the $21^{\text {st }}$ century skills among the students [4]. The steps in our Student Learning Centric an Analogous Learning methodology are given below.

\section{Define Learning Objectives and respective Learning} Outcomes.

2. Explain a real time scenario that can best fit the concept of learning objectives.

3. Probe the questions to the students that bring out points to be emphasized from that real time example that are needed to meet the learning objectives.

4. Using the points inferred from the example introduce the concept to be taught.

5. Evaluate the level of students understanding and take necessary steps to further enhance the level of understanding and involvement.

\section{1: Experiment}

We experimented with this methodology by considering the following learning objective, learning outcomes and assessment objectives.

Learning Objective: To be able to explain the 7 layers of the OSI Model.

Learning Outcome: 1. Students must be able to understand and apply the OSI model layers.

\section{Assessment objectives:}

i) To recognize the number of students who understands the 7 layers of the OSI Model.

ii) To recognize the number of students who work creatively in explaining the OSI model.

iii) To categorize the creative students into one cluster and the students who just understands the OSI model 7 layers into another cluster.

iv)The creative clustered group guides the non-creative clustered group in enriching the applicative thinking and to awaken the joy of creative thinking

v) To innovate a new Active E-Learning technology that demonstrates the 7 layers of OSI Model.

In our example, the imagination starts with a bike racing game named as "Bike Scurry" which has the following rules and regulations.

\section{BIKE SCURRY Safety First Destination Next Game \\ Rules:}

1. The player should start his bike only at the boarding point given to him.

2. The player must wear a helmet and protective suit before he/she drives a bike else he/she will be disqualified. Then the player will be given a packet to handover at the destination point.

3. The destination location is sent via message to the player and needs to follow the Google map to find the route.

4. At every junction, the player has to collect a packet wrapper and needs to wrap the packet. The packet must be delivered with all wrappers in proper format without any damage/loss.

5. At every junction there is a Policeman to check whether the player is following all the rules and regulations with the help of CCTV cameras during the journey.

6. Before reaching the destination point, the player has to give the final packet to a person carrying INDIA map in his hands and must submit all the details of packet carried by him during the race and take the details of the destination point.

7. When the player reaches the destination point all the details of his/her journey with intermediate stages, journey time and information carried via packets will be displayed 
in predefined format and will be used to announce the winner of the race.

The ideology of this game is to make all the players understand that at every stage there is new learning points (rules) added to the previous ones. This simulates with the additional information added at each layer in the OSI model. The first 3 layers of the OSI model and its equivalent steps in Bike Scurry race game are shown in the Figure 4

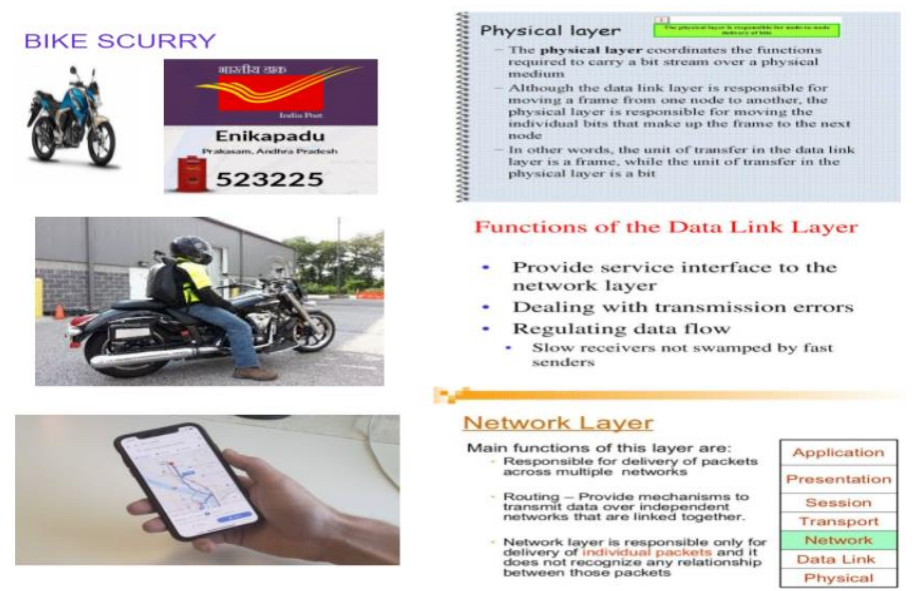

Fig4: Bike Scurry 3 equivalent steps demonstrating physical, datalink and network layers in OSI model.

3.1.1. Physical Layer: Bike is at the boarding point location and physical medium is road. This correlates to the functionality of physical layer to carry the information in the form of bits from source to destination. The physical layer deals with the bit-level transmission between different devices and supports electrical or mechanical interfaces connecting to the physical medium for synchronous as well as asynchronous communication.

3.1.2. Data link Layer: The player must wear helmet and suit in order to drive the bike to ensure him safety. Similarly, data link layer ensures data safety by combining the data bits into frames and adds information to detect and control transmission errors. The three main functions of the data link layer are to deal with transmission errors, regulate the flow of data, and provide a well-defined interface to the network layer. Here regulation and a welldefined interface is analogous helmet and suit given to the player in the second level of the game.

3.1.3. Network Layer: The GPRS enabled tracking location facility is given along with the Bike to the player to track his current location. The destination location is given to the player via SMS time to time in the form of Google map to know the route of his travel. This brings out the functionalities of network layer. Network layer provides data routing path for packets within the network. Data is transferred in the form of packets via logical network paths in an ordered format controlled by the network layer. Similarly, a packet collected by the player at every junction point and handed over at next junction resembles the ordered format of packet delivery in the network layer. The transport layer and session layer functionalities are shown in Fig 5.

3.1.4.Transport Layer: To drive the bike in the traffic or near the signals, the pre-defined rules of the competition play a major role in controlling the bike. The packets are to be delivered by the player without any loss following the rules that resembles packet delivery in the transport layer subject to the protocols. The transport layer provides logical communication between application processes running on different hosts within a layered architecture of protocols and other network components. In the game example, the player should communicate with a person to collect the packet and follow the rules that resemble protocols in transport layer.

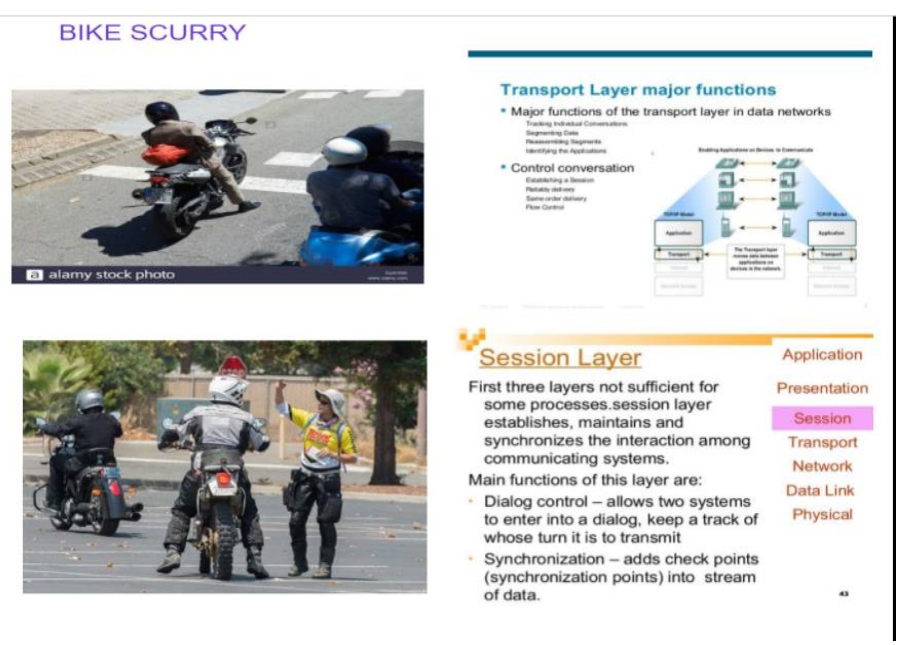

Fig 5:Bike Scurry $4^{\text {th }} \& 5^{\text {th }}$ steps resembling transport and session layers

3.1.4.Transport Layer: To drive the bike in the traffic or near the signals, the pre-defined rules of the competition play a major role in controlling the bike. The packets are to be delivered by the player without any loss following the rules that resembles packet delivery in the transport layer subject to the protocols. The transport layer provides logical communication between application processes running on different hosts within a layered architecture of protocols and other network components. In the game example, the player should communicate with a person to collect the packet and follow the rules that resemble protocols in transport layer.

3.1.5. Session Layer: The session layer establishes, controls, and ends sessions occurring between communicative applications. In the game there are Police personnel at every junction to monitor and coordinate the players. The top two layers presentation layer and application layer functionalities are shown in Fig 6.

3.1.6. Presentation Layer: The presentation layer acts as a translator between the application and the network, mainly addressing the syntax representation of user information that is providing formatted representations and translation data services. Data compression, decompression, encryption, decryption are done in this layer. 
Here in the game the person carrying INDIA flag acts as a translator in making the player to know his destination and in collection of the packet with its wrappers carried by the player during his journey.

3.1.7. Application Layer: It consists of protocols that focus on process-to-process communication across an IP network and provides a firm communication interface and end-user services.

In the game at the destination after parking the Bike at the allotted spot, the details of the packet along with its wrappers, time and speed between each junction and at checkpoints will be displayed in predefined format for each player. These details of all the players are used to announce the winner of the race. The whole gaming scenario must be explained in the classroom by using the analogous teaching mechanism where every student will be able to understand the topic in depth and width.

The theme of the above teaching learning program is to make and recreate the creative minds in the classroom.

BIKE SCURRY
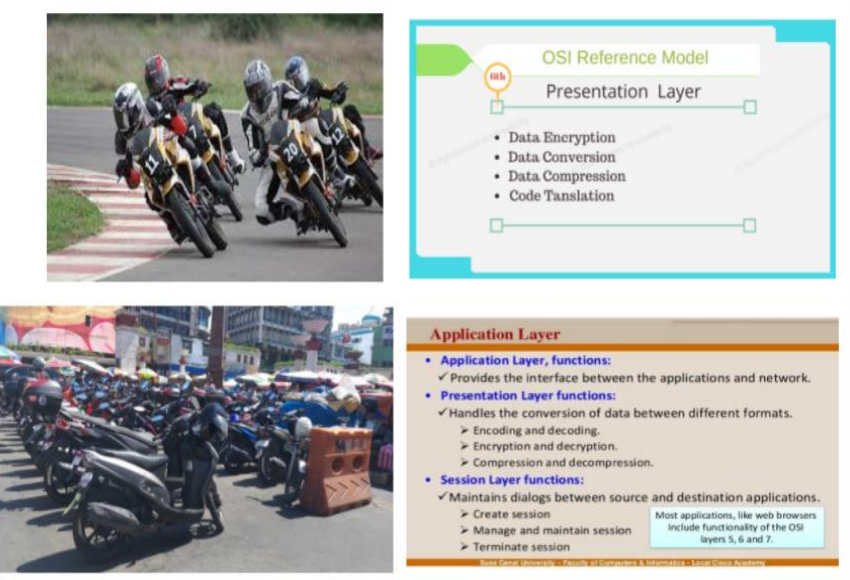

Fig6: Bike Scurry final steps resembling the presentation and application layers.

This method of Analogous learning will help the student in involved and applicative learning. The Best brains of the nation lie in the last benches of a classroom", in the words of A.P.J. Abdul Kalam and this approach might grab the attention of the last bench students.

\section{Results and Assessment of SCL methodology}

In order to evaluate the understanding level of students and measure the effectiveness of our Analogous Learning technique, we defined the assessment objectives as mentioned in the Section 3. The concept of OSI layers was explained to 4 sections of second year B.Tech students, 2 sections without Bike Scurry example and for other 2 sections with Bike Scurry example. After explanation by the teacher, the students were paired and each student was asked to explain the concept to the other student (JIGSAW) approach They were also given an activity to identify the specific jobs done at each layer while they send a mail to his/her friend and get reply mail. After the explanation a survey was conducted for the participants on their ability to understand the functionality of each layer, applying the knowledge to identify the specific jobs done at each layer when a mail is being sent from source to destination system and also on the pleasure of learning the topic.

The survey questionnaire consisted of the following questions.

1. Do you understand the topic?

2. Could you explain and elaborate the topic?

3. How is your experience in imagining and playing the Bike Scurry Race game?

4. Explain the specific jobs done at each layer while sending and receiving a mail.

The student strength in A, B, C and D sections are 67, 65, 60 and 66 respectively. All the sections are approximately equal in terms of their academics, grasping and creative skills.

For the first two sections first two survey questions were given and for the other two sections all the 4 survey questions were given. The result analysis starts with comparison of percentage of students who understood the OSI layers functionality with and without Bike Scurry example and is shown in Table 1.

\begin{tabular}{|l|l|l|l|l|}
\hline $\begin{array}{l}\text { Number of } \\
\text { Students in } \\
\text { Section }\end{array}$ & $\begin{array}{l}\text { Number of } \\
\text { students } \\
\text { understand } \\
\text { the topic }\end{array}$ & $\begin{array}{l}\text { Section } \\
\text { Scurry } \\
\text { Analogy } \\
\text { Given }\end{array}$ & $\begin{array}{l}\text { Percentage } \\
\text { of students } \\
\text { who } \\
\text { understood } \\
\text { the topic }\end{array}$ \\
\hline 67 & 37 & A & NO & $55.6 \%$ \\
\hline 65 & 38 & B & NO & $58.6 \%$ \\
\hline 60 & 44 & C & YES & $73.2 \%$ \\
\hline 66 & 48 & D & YES & $72.7 \%$ \\
\hline
\end{tabular}

Table 1: Percentage of Students who understood the topic

Figure 7 and 8 show the pie chart of the student's clarity without and with Bike Scurry Example. It clearly shows that the percentage of students who understood the topic is 15-20\% higher with Analogous Learning.

1.Do you understand the topic
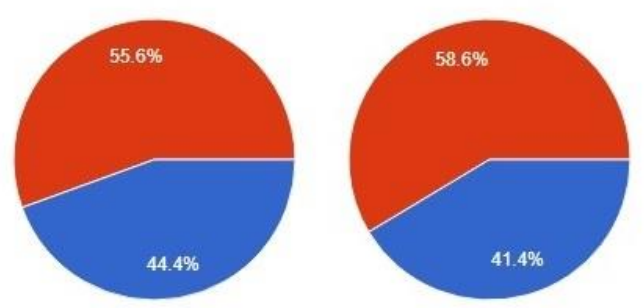

Yes

No

Paybe

Fig 7: A \& B Sections Student's clarity without Bike Scurry 


\section{Do you understand the topic}
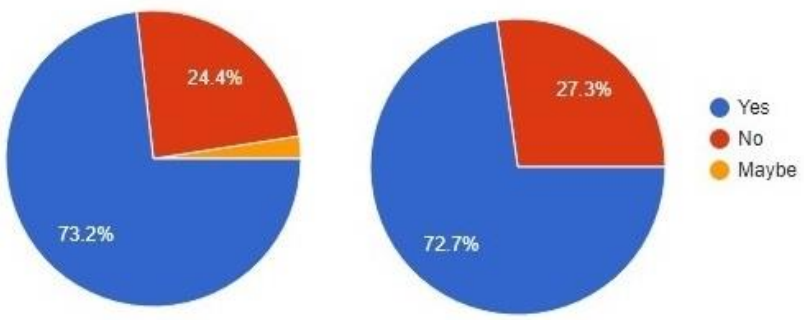

Fig 8: C \& D Sections clarity with Bike Scurry

Figure 9 shows C \& D section student's clarity after JIGSAW activity among the student's of the same section. It shows a further improvement of 10-12\% after the JIGSAW active learning activity.

From the two tables $1 \& 2$ it is evident that the clustered active learning students group are mostly visual learners because they can understand the topic with the gaming scenario in better manner.

\section{Could you explain and elaborate the topic}
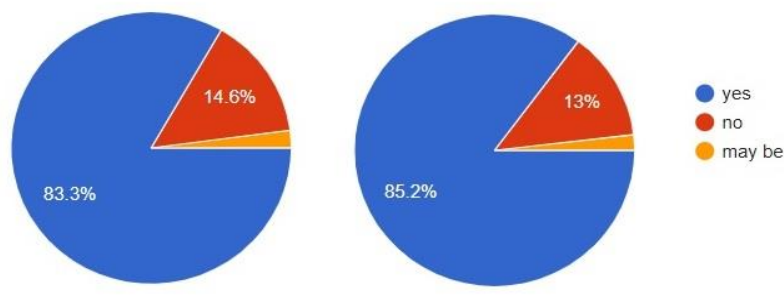

Fig 9: C \& D Section students experience in Elaborating the topic

A JIGSAW approach was further carried out among the sections taught with and without Game Scurry example. That i,s a student of Section A is paired with a student of Section C and similarly a student of Section B is paired with a student of section $\mathrm{D}$. Then the survey comprising all the four questions was collected from Sections A \& B. Figure10 shows the improvement in student's learning experience and joy with Analogous Learning based on Bike Scurry Example and also JIGSAW activity. The Overall understanding level of students has been evaluated based on the following attributes:

1. Enthusiasm

2. Willingness

3. Learning Aptitude

4. Questioning.

5. Creative thinking.
A Questionnaire containing the above attributes is given to them and the overall results are tabulated in Table 2

\begin{tabular}{|l|l|l|l|l|}
\hline S.No & Section & $\begin{array}{l}\text { Number } \\
\text { of } \\
\text { students }\end{array}$ & & \multicolumn{2}{|l|}{ Percentage of students } \\
\cline { 4 - 5 } & & & $\begin{array}{l}\text { Without } \\
\text { JIGSAW }\end{array}$ & $\begin{array}{l}\text { With } \\
\text { JIGSAW }\end{array}$ \\
\hline 1 & & & 55.6 & 70.2 \\
\hline 2 & $\mathrm{~B}$ & 67 & 58.6 & 72.3 \\
\hline 3 & $\mathrm{C}$ & 65 & 73.2 & 83.3 \\
\hline 4 & $\mathrm{D}$ & 66 & 72.7 & 85.2 \\
\hline
\end{tabular}

Table 2: Comparison of Percentage of students who understood before and after JIGSAW

From Figure 10 it is evident that more than $80 \%$ of students have shown interest and willingness in listening to the topic in Analogous and JIGSAW Active Learning technique.

\section{HOW IS THE GAME LEARNING EXPERIENCE}

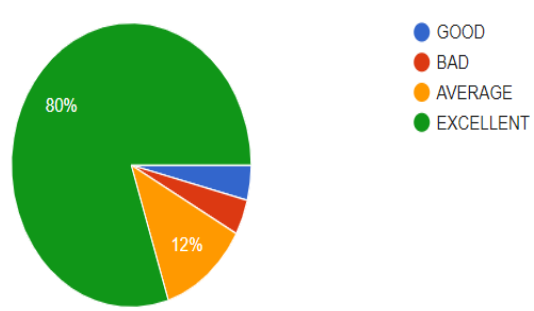

Fig 10: Overall Experience of students with JIGSAW

\section{Conclusion}

This paper emphasises the need for getting insights into learning process coupled with Analogous Learning. The teacher needs to identify and explain more appropriate and interesting example for introducing new concepts. This Student Centric Analogous Learning methodology was implemented to explain OSI Layers, an important topic in Computer Networks with the help of relevant Bike Scurry race example. The effectiveness of learning in the class was assessed with properly defined assessment objectives. The experimental results show that the Analogous Learning technique was more useful in improving comprehensibility, applicability and joy of learning. The efficacy of learning outcome may be further enhanced using the animation and Artificial Intelligence techniques (like using Amazon Alexa) and ultimately in grabbing the interest of more number of students in the class. 


\section{References}

[1].G.D. Catalano, K.C. Catalano., "Transformation: from Teacher-Centered to Student-Centered Engineering Education" Proceedings frontiers in education 1997 27th annual conference. teaching and learning in an era of change.

[2]. Amal Abdel SattartWafaLabib "Active learning in Engineering Education: Teaching Strategies and Methods of overcoming challenges" Proceedings of $20198^{\text {th }}$ international conference on Educational and Information technology pages 255-261

[3]. Ricardo Ambrocio Ramírez-Mendoza, Lorena A. CruzMatus, Elisa Vazquez," Towards a disruptive active learning engineering education" IEEE Global Engineering ISSN: 2165-9567

[4]. Divya Asok ; A.M. Abirami ; Nisha Angeline," Active Learning Environment for Achieving Higher-Order Thinking Skills in Engineering Education” 2016 IEEE 4th International Conference on MOOCs, Innovation and Technology in Education (MITE)

ISBN: 978-1-5090-1062-2

[5]. Magana, Alejandra J.; Vieira, Camilo; Boutin, Mireille," Characterizing Engineering Learners' Preferences for Active and Passive Learning Methods" IEEE Transactions on Education, v61 1 p46-54 Feb 2018

[6].Babatunji Adedeji, Mohammad Mizanur Rahman" Innovative Teaching Methods and Entrepreneurship Education: A Review of Literature" Journal of Business Economics and Management Volume 10, Issue 1 ISSN: 2395-2210

[7]. Zaidatol Akmaliah Lope Pihi, Keetanjaly Arivayagan," Teaching Strategies as a Predictor of Entrepreneul Mindset: Implications for Innovative Teaching Strategies" International Journal of Innovative Research in Technology \& Science ISSN:2321-1156

[8].MunaAljohani,"Innovation and Entrepreneurship Integration in Education. Ohaio State Model" International Journal of Teaching and Education vol. 3, no. 3 / 2015 